\title{
Quantitative evaluation and significance of ultrasound in bronchoalveolar lavage for lung consolidation in children with severe mycoplasma pneumonia
}

\author{
Shuo Li ${ }^{1}$, Qi-Li Zhang ${ }^{1}$, Rui-Jun Guo ${ }^{1}$, Xiu-Zhang Lv $^{2}$, Xue Yang ${ }^{3}$ \\ ${ }^{1}$ Department of Ultrasound Medicine, Beijing Chaoyang Hospital, Capital Medical University, Beijing, China; ${ }^{2}$ Department of Echocardiography, \\ Beijing Chaoyang Hospital, Capital Medical University, Beijing, China; ${ }^{3}$ Department of Pediatrics, Beijing Chaoyang Hospital, Capital Medical \\ University, Beijing, China \\ Contributions: (I) Conception and design: S Li; (II) Administrative support: XZ Lv, X Yang; (III) Provision of study materials or patients: S Li, QL \\ Zhang; (IV) Collection and assembly of data: S Li, QL Zhang, RJ Guo; (V) Data analysis and interpretation: S Li, RJ Guo; (VI) Manuscript writing: \\ All authors; (VII) Final approval of manuscript: All authors. \\ Correspondence to: Xiu-Zhang Lv, PhD. Department of Echocardiography, Beijing Chaoyang Hospital, Capital Medical University, North Road 8, \\ Gongti, Chaoyang, Beijing 100020, China. Email: 276264624@qq.com; Xue Yang, MD. Department of Pediatrics, Beijing Chaoyang Hospital, \\ Capital Medical University, North Road 8, Gongti, Chaoyang, Beijing 100020, China. Email: ziien@hotmail.com.
}

Background: The aim of this study was to evaluate the effect of lung ultrasound on bronchoalveolar lavage in the treatment of mycoplasma pneumonia in children.

Methods: Thirty children with mycoplasma pneumonia who were treated with medication and bronchial lavage were selected. We collected the results of laboratory and imaging examinations after admission, and the changes in lung consolidation area were examined by ultrasound before, immediately upon completion, and at 1,3, and 7 days after bronchoalveolar lavage. The effective factors affecting bronchial lavage were analyzed through logistic regression.

Results: Univariate regression analysis showed significant differences in the lung consolidation area at admission, before, immediately upon completion, and at 1 and 7 days after lavage, as well as in the disease course and atelectasis (or not). Multivariate analysis showed that there were statistical differences in the lung consolidation area at admission, atelectasis (or not), and disease course: lung consolidation area at admission [odds ratio (OR): 2.31512, 95\% confidence interval (CI): (0.00182, 0.031775), P=0.029474], atelectasis [OR: 2.695742, 95\% CI: (0.079281, 0.597218), P=0.012629], and disease course [OR: $-2.43347,95 \% \mathrm{CI}$ : $(-0.02568,-0.00211), \mathrm{P}=0.022773]$.

Conclusions: Lung ultrasound can evaluate the effect of bronchial lavage through lung consolidation and atelectasis, which can provide a reference for clinical treatment.

Keywords: Lung ultrasound; bronchoalveolar lavage; mycoplasma pneumonia in children; lung consolidation

Submitted Jul 15, 2021. Accepted for publication Sep 07, 2021.

doi: 10.21037/tp-21-381

View this article at: https://dx.doi.org/10.21037/tp-21-381

\section{Introduction}

Statistically, mycoplasma pneumoniae (MP) accounts for $15-20 \%$ of common pathogens of community-acquired pneumonia (CAP) and $40 \%$ of common pathogens of CAP in children $(1,2)$. Clearly, the mycoplasma was the main cause leading to MP and pediatric mycoplasma pneumonia occupies a dominant position in pediatric CAP. As the immune system of children is not fully developed and the elastic tissue of the lungs is in the development stage, their respiratory system defense function is poor, and thus pathogenic bacteria can easily enter the respiratory tract and cause pulmonary infection (3). If the illness cannot 
be effectively controlled, it will cause focal diffusion, and even lead to atelectasis, pulmonary infarction, and systemic diseases (including the nervous system, circulatory system, etc.). It may even result in severe MP pneumonia (SMPP) $(4,5)$, and ultimately lead to an increase in child mortality rates.

In recent years, the popularity of alveolar lavage with fiberoptic bronchoscope has gradually increased. This technique has characteristics including wide field of vision, high definition, little stimulation, etc., and can clearly display mucosal lesions and tube wall movement under the microscope. Many studies have shown that fiberoptic bronchoscope alveolar lavage is significantly better than drug therapy alone for the treatment of SMPP, when there is a large area of consolidation in the lung, fiberoptic bronchoscopy lavage is the first choice (3). Although fiberoptic bronchoscopic alveolar lavage is a successful procedure, it is potentially risky and can cause significant irritation and injury to children. In addition, fiberoptic bronchoscopy involves high-level technical requirements. In the past, CT or X-ray was used for adjuvant treatment. The innovation of this paper is to use ultrasound for localization and curative effect evaluation (6). Lung ultrasound, as a portable, radiation-free, and portable diagnostic imaging tool, is crucial for determining the effect of bronchofiberoptic alveolar lavage in the treatment of SMPP (6).

The purpose of this study was to quantitatively evaluate the effect of bronchoalveolar lavage on children with SMPP atelectasis, and to determine the best time for treatment using this technology by monitoring the efficacy of ultrasound, to effectively address the clinical needs and reduce the hospitalization costs of patients. We present the following article in accordance with the STARD reporting checklist (available at https://dx.doi.org/10.21037/tp-21381).

\section{Methods}

\section{General information}

A total of 30 children with SMPP (including 16 males and 14 females, aged 6-13 years, with an average age of $9.27 \pm 2.07$ years) from the Department of Pediatric Surgery of Beijing Chaoyang Hospital between September 2019 and March 2020 were included. The patients' conditions upon admission were collected, and laboratory examinations [neutrophil\% (NE\%), lymphocyte\% (LY\%), neutrophilic stab granulocyte (NST), C-reaction protein (CRP), and procalcitonin (PCT)], and imaging examinations (chest CT, X-ray) were performed after admission. All procedures performed in this study involving human participants were in accordance with the Declaration of Helsinki (as revised in 2013). The study was approved by the Ethics Committee of Beijing Chaoyang Hospital (No.: 2019-19) and informed consent was taken from all the patients.

\section{Inclusion criteria}

All included children met the diagnostic criteria of the Expert consensus on diagnosis and treatment of mycoplasma pneumoniae pneumonia in children (2015 edition) (7) and had indications for bronchofibroscopy alveolar lavage (8): poor efficacy of routine treatment, and improvement of pulmonary signs was not obvious. Also, the patients had obvious signs and symptoms of airway obstruction (such as reduction or disappearance of breathing sounds, tubular breathing sounds, and repeated fixation of wheezing sounds). Lung ultrasound was used to evaluate the scope of lung consolidation and atelectasis.

\section{Exclusion criteria}

(I) Patients with tuberculosis, bronchial asthma, bronchial foreign body;

(II) Patients with congenital heart disease and arrhythmia;

(III) Patients with severe extrapulmonary complications (severe exudative erythema pleomorphic, acute nephritis syndrome, etc.);

(IV) Children with backward motor development, severe malnutrition, low immune function, and repeated hospitalization due to pneumonia within 1 month;

(V) Patients with incomplete clinical data, and those transferred another hospital due to a variety of reasons.

\section{Instruments and methods}

The color Doppler ultrasonography instrument (Canon, Japan) was used for pulmonary ultrasound examination, and a higher linear probe (7.5-10 MHz) (Canon, Japan) was used for superficial pleural and subpleural lesions. A lower frequency convex probe (2-5 MHz) (Canon, Japan) was used for deep lesions and body fat person. The children were placed in supine, semi-recumbent, lateral, prone, or sitting positions in a quiet state. The probe was placed 

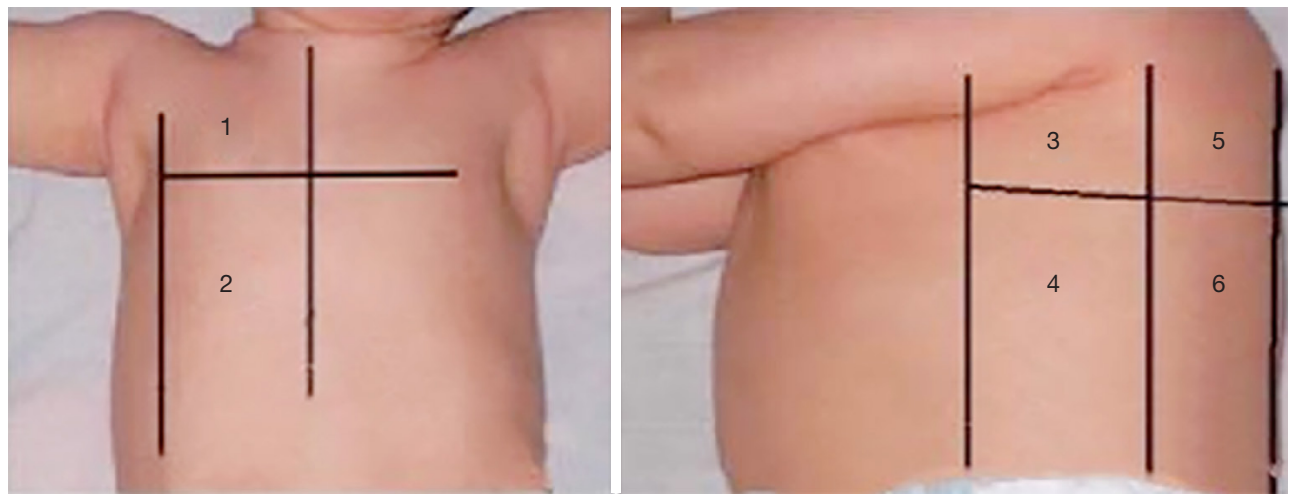

Figure 1 Children's lungs are drawn on the body surface. The zones were denoted as R1-6 (zones 1-6 of the right thoracic cavity) and zone L1-6 (zones 1-6 of the left thoracic cavity). This image is published with the patient's consent.

either vertically or horizontally with ribs, with axillary front, axillary line is bounded to the lungs is divided into front, side and back three areas, nipple plane as the upper and lower boundary. That is, the left/right lungs were divided into six areas, with a total of 12 bilateral areas for a comprehensive scan.

As shown in Figure 1, the zones were denoted as R1-6 (zones 1-6 of the right thoracic cavity) and zone L1-6 (zones 1-6 of the left thoracic cavity).

Fiberoptic bronchoscopy alveolar lavage was performed using an electronic bronchoscope (Olympus, Japan). Children were placed in the supine position and a local surface anesthesia compound was used for conscious sedation. Subsequently, an intraoperative side nasal cavity was used to insert the oxygen pipe when administering oxygen inhalation through the nasal passages, Subsequently, an intraoperative side nasal cavity was used to: (I) insert the oxygen pipe when administering oxygen inhalation through the nasal passages (through the nose into the mirror); (II) observe the glottis, trachea, lung and each lobe bronchus; (III) reach the lesions in the lung segment (which could be directly delivered to the results of the ultrasound lung lesions lung segment); and (IV) suction secretions. Next, under direct bronchoscope vision, the disaffected lung segments were lavage with $37^{\circ} \mathrm{C}$ normal saline $(10-15 \mathrm{~mL}$ each time, total amount $\leq 5 \mathrm{~mL} / \mathrm{kg}$ ). After each lavage, the alveolar lavage fluid was subjected to negative pressure suction (negative pressure $100-200 \mathrm{mmHg}$ ), and the alveolar lavage fluid was examined. This was repeated 2 3 times. Also, respiration, heart rate, complexion, and blood oxygen saturation were observed.

\section{Observation indicators}

Pulmonary ultrasound primarily observed the pleural line, A-line, B-line, bronchial sign, lung consolidation, and pleural effusion. Lung ultrasonography was performed on each child after admission, as well as before, immediately upon completion, and at 1,3 , and 7 days after lavage. We recorded the area of pulmonary consolidation and whether there was a dynamic or static bronchial air sign. The area consolidation zone changes after irrigation were compared (1-, 3-, and 7-day difference).

Evaluation of the curative effect after lavage was based on the research criteria reported in previous literature $(9,10)$ : (I) cure: child's clinical symptoms and signs completely disappeared after treatment, and the location and range of the atelectasis marked by ultrasound before treatment showed that the area of atelectasis decreased by more than $90 \%$ after ultrasound reexamination after lavage treatment; (II) significant effect: the child's clinical symptoms and signs were improved after treatment, and the atelectasis area was reduced by $60-90 \%$; (III) effective: the child's clinical symptoms and signs were improved after treatment, and the atelectasis area was reduced by 30-59\%; and (IV) no effect: the child's clinical symptoms and signs did not improve after treatment, and the atelectasis area decreased by less than $30 \%$. The total effective rate was calculated as follows: total effective rate $=($ cured cases + effective cases + effective cases $) /$ total number of cases $\times 100 \%$. All sonograms were performed by two experienced sonographers, and the results were mutually validated. 


\section{Statistical analysis}

Statistical software (SPSS, version 23.0; SPSS) was used for statistical analysis of the data. Measurement data were expressed as mean \pm standard deviation $(\bar{x} \pm s)$. The homogeneity of variance test was performed between groups first; the independent sample $t$-test was used for homogeneity of variance, and the approximate $t$-test was used for heterogeneity of variance. Enumeration data were represented as rate (\%), and the Fisher's exact probability test was used. $\mathrm{P}<0.05$ was considered to indicate a statistically significant difference. Univariate and multivariate logistic regression were used to analyze the effective factors of bronchial lavage, and the data were presented as odds ratios (ORs) and the corresponding 95\% confidence interval (CI). Whether the patient was effective or not was used as an independent variable $(0=$ ineffective, $1=$ effective).

The discovery of area changes before and after irrigation was the main factor of logistic regression. We adjusted for the following factors: gender (logistic regression value: 0 for males, 1 for females), age, NE\%, LN\%, NST, CRP, PCT, duration of disease, and presence of atelectasis, as independent variables to establish a regression model (inclusion criteria: $\mathrm{P}<0.05$, exclusion criteria: $\mathrm{P}>0.10$ ). The remaining logistic regression values were all the measured values themselves. The regression $\mathrm{P}$ value was used to judge whether the prediction was effective $(\mathrm{P}>0.5$ was invalid, and $\mathrm{P} \leq 0.5$ was effective). The regression parameters were estimated by the Wald $\chi^{2}$ test. The likelihood ratio (OR value) was used to test the fit of the evaluation model (OR value $>1$ was a risk factor).

\section{Results}

\section{Clinical study results}

Among the 30 SMPP children who had received bronchial lavage, the average duration of disease was $21.6 \pm 11.5$ days. All the children were treated with drugs combined with bronchial lavage and were finally cured and discharged. The clinical manifestations of the children were fever, cough, runny nose, and yellow nasal.

Bronchial lavage was effective in 20 cases and the improvement rate was $66.7 \%$. The inefficiency rate was $33.3 \%$ in 10 cases. After admission, the laboratory indexes increased to varying degrees, but the effectiveness of bronchial lavage was not relevant. After admission, the mean values of NE\%, LY\%, NST, CRP, and PTC were
$56.5 \%$ (57.7\% effective, $54 \%$ ineffective, $\mathrm{P}=0.507$ ), $32.8 \%$ (32.1\% effective, $35.6 \%$ ineffective, $\mathrm{P}=0.694$ ), $5 \%$ (4.6\% effective), $5.1 \%$ (6.5\% effective, $2.4 \%$ ineffective, $\mathrm{P}=0.694$ ), and $0.22 \%(0.2 \%$ effective, $0.2 \%$ ineffective, $\mathrm{P}=0.694)$, respectively (Table $\mathrm{S} 1$ ).

\section{Pulmonary ultrasound characteristics of children with SMPP}

All 30 patients underwent at least nine pulmonary ultrasonography examinations, and the results are shown in Table 1. Twenty-seven patients (90.0\%) had an abnormal pleural line (Figure 2A). All 30 cases (100.0\%) had scattered B-lines (which could be counted) and fused B-lines (which were difficult to distinguish and count) (Figure $2 B$ ). Furthermore, all 30 patients $(100.0 \%)$ presented lung consolidation of varying degrees (ultrasound images showed liver-like changes in lung tissue, possibly accompanied by dynamic bronchial air sign) (Figure 2C). Eighteen patients $(60.0 \%)$ had atelectasis (Figure 2D,2E) with a large and regular consolidation range, often accompanied by a static air bronchial sign and abundant blood flow, suggesting reversible atelectasis without irreversible changes (such as necrosis). Sixteen patients (53.3\%) had pleural effusion (Figure $2 F$ ), with an average effusion depth of $1.0 \pm 1.5 \mathrm{~cm}$.

\section{Logistic analysis results}

The study period was from September 2019 to March 2020. The results of univariate and multivariate logistic analysis are shown in Table 2. Univariate analysis showed that there were statistically significant differences in the changes of pulmonary consolidation area at admission, before and after lavage, atelectasis, and duration of disease. Multivariate analysis showed that there were statistically significant differences in pulmonary consolidation, atelectasis, and duration of disease at admission. The likelihood ratio test $\left(\chi^{2}=7.829531, \mathrm{P}=0.000172\right)$ indicated that the model was statistically significant. The regression model was used to predict the effectiveness of bronchial lavage in children with a regression $\mathrm{P}$ value; if the prediction was invalid when the regression $\mathrm{P}$ value was $>0.5$ and effective when $\mathrm{P} \leq 0.5$, the prediction accuracy reached $90.5 \%$. Area under the receiver operating characteristic (ROC) curve was 0.8825 (Figure 3). The course of disease in 20 patients with effective broncholavage was $13 \pm 2$ days, and $23 \pm 6$ days in 10 patients with invalid broncholavage. The two groups were compared using the $t$-test, and the statistical results were: $\mathrm{t}=9.766$, 
Table 1 Pulmonary ultrasound characteristics of children with SMPP

\begin{tabular}{|c|c|c|}
\hline Positive cases & Ultrasound findings & Characteristics \\
\hline 30 (100.0\%) & Scattered B lines & $\begin{array}{l}\text { From the pleural line, which extends vertically and radiates to the depth of the lung, these could } \\
\text { be distinguished and counted }\end{array}$ \\
\hline 30 (100.0\%) & Confluent B lines & $\begin{array}{l}\text { The dense B-lines that can be found in all intercostal spaces were difficult to distinguish and } \\
\text { count }\end{array}$ \\
\hline $18(60.0 \%)$ & Atelectasis & $\begin{array}{l}\text { Ultrasound showed liver-like changes in lung tissue, with a possible bronchial air sign, and the } \\
\text { bronchial air sign was static }\end{array}$ \\
\hline $16(53.3 \%)$ & Pleural effusion & The average depth $1.0 \pm 1.5 \mathrm{~cm}$ \\
\hline
\end{tabular}

SMPP, severe mycoplasma pneumoniae pneumonia.
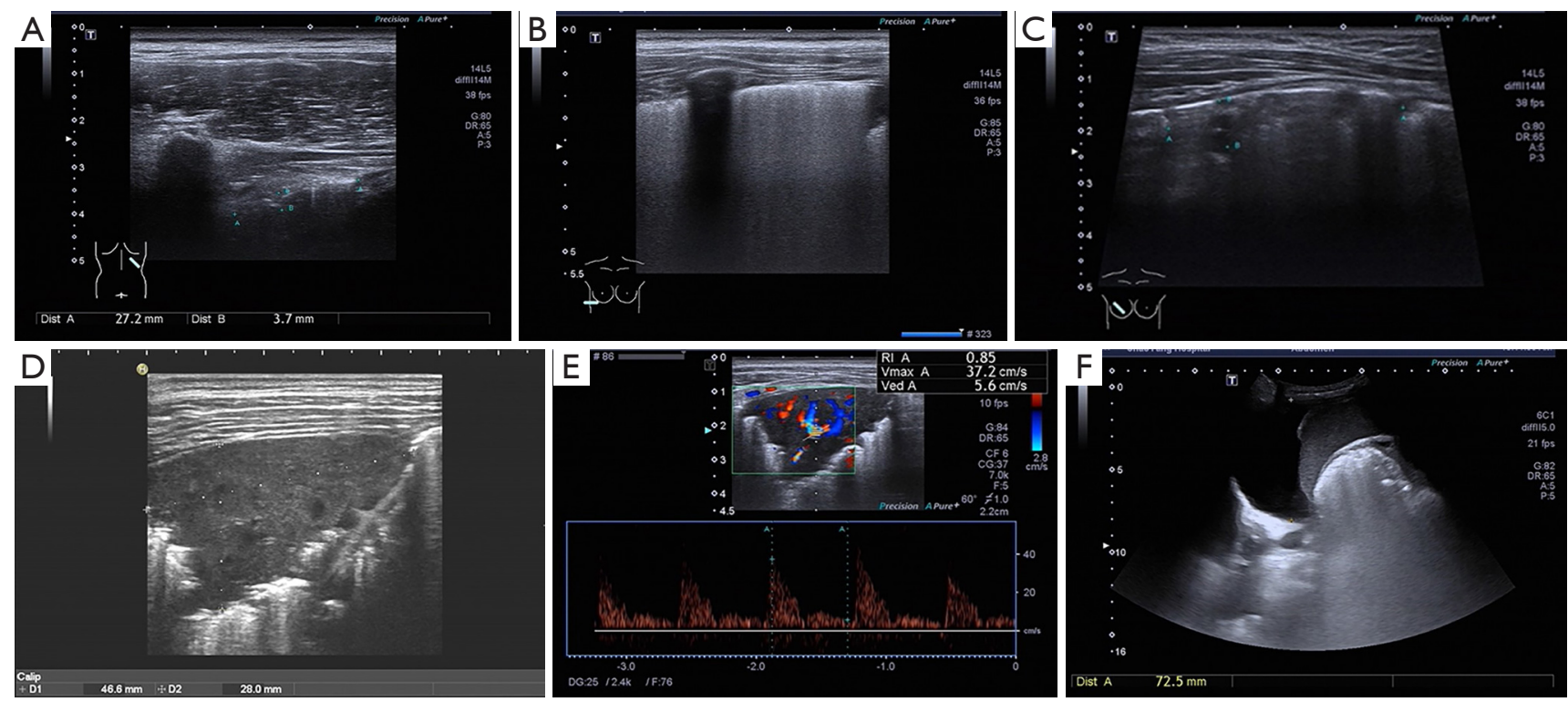

Figure 2 Ultrasonography of lung in children with SMPP. (A) The right pleural line was thickened, blurred, and discontinuous after posterior chest wall exploration; (B) female, 9 years old, anterior chest wall exploration revealed fusion of the B-line and AIS in the right lower lung. The probe as scanned vertically with the ribs; if the entire intercostal space showed B-line fusion, it was difficult to distinguish and count; if the sound shadow of the ribs was still clearly displayed, this dense B-line was called a fusion B-line. When there were more than two consecutive fused B-lines in the intercostal spaces in any scanning area, it as regarded as AIS; (C) male, 6 years old, 7 days after fiberoptic bronchoscopic lavage, the area of consolidation in the right lower lung was significantly reduced on anterior chest wall exploration, and dynamic bronchial signs were observed on real-time ultrasound exploration; (D) female, 10 years old, lung consolidation. Lung tissue showed hepatoid degeneration. Under real-time ultrasound, a static bronchial air sign could be seen in the lung tissue. A fragment sign could be observed in the transition zone from the consolidated lung tissue to the aerated lung tissue; (E) CDFI showed rich blood flow signals in the condensed lung tissue, PW could detect the blood flow spectrum of the high resistance artery, RI 0.85; (F) female, 6 years old, with large pleural effusion on the left side and compressed lung tissue nearby. SMPP, severe mycoplasma pneumoniae pneumonia; AIS, alveolar-interstitial syndrome; CDFI, color Doppler flow imaging; PW, pulse Doppler; RI, resistance index. 
Table 2 Logistic analysis results

\begin{tabular}{|c|c|c|c|c|c|c|c|c|}
\hline \multirow{2}{*}{ Contents } & \multicolumn{4}{|c|}{ Univariate } & \multicolumn{4}{|c|}{ Multivariate } \\
\hline & OR & Lower & Upper & $\mathrm{P}$ & OR & Lower & Upper & $P$ \\
\hline Lung consolidation area at admission & 2.902 & 0.004 & 0.023 & 0.007 & 2.315 & 0.002 & 0.032 & 0.029 \\
\hline Lung consolidation area before lavage & 3.381 & 0.006 & 0.026 & 0.002 & -0.406 & -0.034 & 0.023 & 0.689 \\
\hline Area of lung consolidation 1 day after lavage & 1.972 & -0.001 & 0.027 & 0.059 & -0.417 & -0.025 & 0.016 & 0.681 \\
\hline Area of lung consolidation 3 days after lavage & 0.616 & -0.012 & 0.024 & 0.543 & & & & \\
\hline Area of lung consolidation 7 days after lavage & -1.80 & -0.047 & 0.003 & 0.083 & -1.957 & -0.035 & 0.001 & 0.064 \\
\hline
\end{tabular}

OR, odds ratio; $\mathrm{Cl}$, confidence interval.

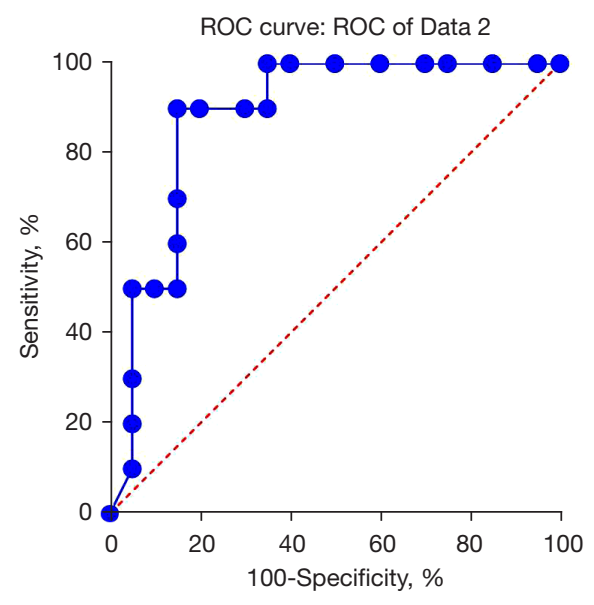

Figure 3 ROC curve of lung consolidation area at admission (area: $0.8825,95 \%$ CI: 0.7592 to $1.000, \mathrm{P}=0.0008$ ). ROC, receiver operating characteristic; CI, confidence interval.

$\mathrm{P}<0.0001$, as shown in Figure 4.

\section{Analysis of pulmonary invalid consolidation}

Pulmonary consolidation was present in every SMPP patient who had received bronchial lavage. In clinical practice, the best time for reexamination is still an open question. In this study, we counted a series of changes in lung consolidation area and concluded that the reexamination can display more obvious changes after 14 days of lavage (Figure 5).

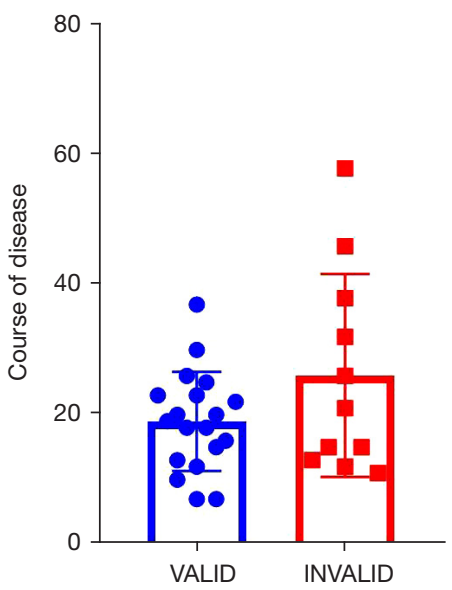

Figure 4 Analysis of the course of disease in SMPP children who had received bronchial lavage between valid and invalid patients (valid: $13 \pm 2$ days; invalid: $23 \pm 6$ days). SMPP, severe mycoplasma pneumoniae pneumonia.

\section{Discussion}

CAP is the most common disease recorded worldwide, with $20-3,000,000$ cases diagnosed annually in the United States (11). In recent years, a growing number of studies have found that MP pneumonia (MPP) exhibits a trend of younger age infection (12). MP is a common pathogen of CAP infection in children. Owing to the under-developed immune system of children, as well as the fact that the 


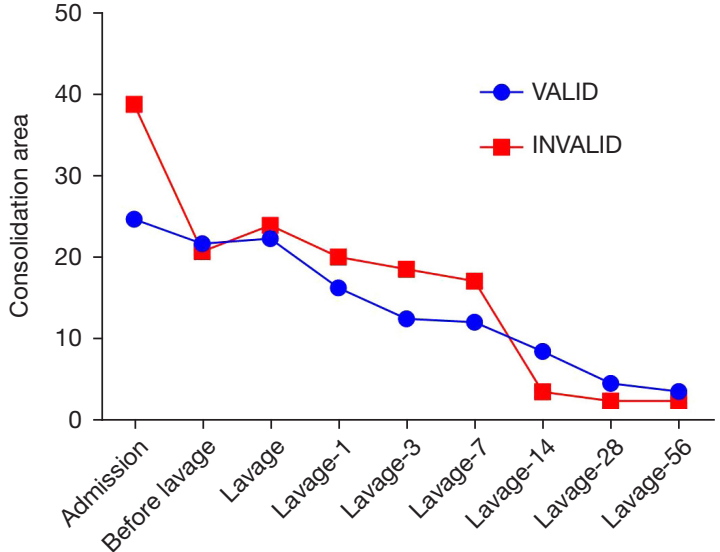

Figure 5 Curve of mean valid and invalid lung consolidation area in children undergoing broncholavage.

elastic tissue of the lungs is in the development stage, and the bronchial cartilage is relatively soft with poor elasticity, the lungs of children are more easily subjected to compression. Also, the respiratory system of children has poor defense function, and pathogenic bacteria can easily enter the respiratory tract and cause pulmonary infection.

In the past, treatment with conventional medicine was used, including spasmolysis, antiasthmatic, expectorant, antibiotics, and so on. In recent years, the mutation rate of MP with high drug resistance has gradually increased (13), and the resistance to macrolide drugs is very high. Hormones and gamma globulin can effectively control fever and excessive inflammation in children with SMPP; however, they cannot clear many bronchial phlegm plugs, as well as necrosis mucosa and plastic phlegm plug obstructions (14). As a result, the disease cannot be effectively controlled, resulting in sequelae such as atelectasis and bronchitis oblans (15), and even serious complications of the nervous, circulation, digestive, and immune systems (16), affecting the physical and mental health of children.

Ultrasound technology has been considered off-limits in the past because the lungs are filled with gas. The depth of ultrasonic pneumonia examination in adults and children is different. Adults mainly use low-frequency probe (2$5 \mathrm{MHz}$ ); children mainly use high-frequency probes (7.5-10 MHz). In recent years, with the continuous study and increased knowledge of lung ultrasound by domestic and foreign ultrasound doctors, lung ultrasound can now be employed with high sensitivity (93.4\%) and specificity $(97.7 \%)$ for the diagnosis of lung diseases in children and newborns, which previously required chest X-ray and CT (11). CT examination can cause more radiation damage to children, so ultrasound has become the main tool for imaging examination of children and newborns with pulmonary diseases. In China, pediatric pulmonary ultrasound has entered a stage of extensive development and mature application. Ultrasound imaging diagnosis of lung diseases such as pneumonia, atelectasis, meconium aspiration syndrome (MAS), and pulmonary hemorrhage has been fully mastered in most children, especially newborns (17). The advantage of ultrasonography is that it has no radiation and can be repeated many times (10-13). We can examine it at multiple time points before and after bronchial lavage; The disadvantage is that in terms of positioning, ultrasound is not as accurate as CT, and cannot provide clinicians with overall spatial perception (15-17).

The purpose of this study was to evaluate the efficacy of bronchofiberoptic alveolar lavage in children with SMPP using ultrasound. Studies have shown that ultrasound can clearly and quantitatively evaluate the effect of bronchofiberoptic alveolar lavage on pulmonary consolidation in children with SMPP. By tracing the area of pulmonary consolidation, the curative effect can be quantitatively evaluated for convenient clinical grading diagnosis and treatment. In this study, it was found that the area of pulmonary consolidation at admission determines the effectiveness of lavage. The larger the area of pulmonary consolidation, the longer the corresponding course of disease, and the more likely it is to be complicated with atelectasis. Thus, we recommend a combination of drug therapy and bronchial lavage, which is consistent with previous reports that lung consolidation affects disease severity.

The incidence of atelectasis in MPP has been reported to be approximately $10 \%$ (18). Previously, the causes of MPP atelectasis were necrosis of the skin cells on the bronchial wall caused by mycoplasma infection, swelling of the bronchial mucosa, smooth muscle spasm, and obstruction of the airway lumen by mucus, cellulose, and broken cells, resulting in occlusion. Moreover, MPP may also be caused by the obstruction of secretions (19). The concepts of atelectasis and pulmonary consolidation are not equal. Pulmonary consolidation is considered when the lung sonogram loses its A- and B-line features and appears like the liver and spleen. An atelectasis is an obstruction or compression of the bronchi, which results in absorption of air in the distal bronchi and alveoli. The volume or air content of one or more lung segments or 
lobes is thereby reduced. Ultrasound may still show signs of pulmonary consolidation. It is often not an independent disease, but a complication of other diseases. Present, the effect of conventional Western medicinal therapy has been markedly weakened, and the alveolar lavage by fiberoptic bronchoscope technique was developed at a historically significant moment. This study also confirmed that bronchial lavage in children with atelectasis can effectively reduce the area of pulmonary consolidation and shorten the course of disease.

The mechanism of alveolar lavage under fiberoptic bronchoscopy is primarily reflected in the following aspects (20): (I) normal saline lavage can promote the local hypertonic state, and then achieve the purpose of eliminating edema, enhancing local blood supply, and effectively improving the symptoms of hypoxia in the body; (II) through lavage treatment, secretions and mucus can be effectively removed, the content of local toxins and inflammatory factors can be reduced, and the damage to the bronchus can be controlled; and (III) it can directly act on lesions, remove mucus plugs and secretions, and improve atelectasis and lung function in a relatively short time. In recent years, fiberoptic bronchoscopy has been widely used due to its characteristics, including a wide field of vision, high definition, and little stimulation. Under the microscope, mucosal lesions and tube wall movement can be clearly displayed. Studies have shown that fiberoptic bronchoscopic alveolar lavage can significantly improve the treatment efficiency and shorten the treatment time of patients with bronchiectasis (21) and pulmonary infection (22). Also, it has been widely recognized that fiberoptic bronchoscopy and alveolar lavage play a significant role in the treatment of infectious atelectasis (23).

Therefore, in the presence of atelectasis in children with SMPP, fiberoptic bronchoscopic alveolar lavage has a marked effect, while pulmonary consolidation caused by pneumonia or pulmonary edema has no obvious effect. The immediate lavage can significantly improve the condition of children with atelectasis on ultrasound examination, and the implementation of lavage can be postponed in cases of non-obstructive pulmonary consolidation, when the area of consolidation after lavage does not change significantly.

This study also found that in addition to removing mucus plugs and secretions during normal saline lavage, there might be residual normal saline after aspiration, leading to no significant reduction or even a slightly increased pulmonary consolidation area monitored by ultrasound immediately after lavage. The atelectasis area decreased to different degrees at 1, 3, and 7 days after lavage, and the area size before and at 7 days after lavage differed in terms of the effectiveness and ineffectiveness of lavage. However, there was no significant difference in the equation. In the paper, only 30 patients were enrolled. These are all cases collected recently, and these results can be obtained. However, further information needs to be collected for verification.

In addition to diagnosing lung diseases, pulmonary ultrasound has also achieved good results in guiding treatment and care of other diseases. For example, under ultrasonic monitoring, bronchoalveolar lavage is used to treat neonatal atelectasis, to guide lavage treatment of severe pneumonia and MAS, to guide the application and withdrawal of ventilator, to guide the application and evaluation of the efficacy of exogenic pulmonary surfactant, and to guide the puncture and drainage of severe pneumothorax and pleural effusion, etc.

\section{Acknowledgments}

Funding: None.

\section{Footnote}

Reporting Checklist: The authors have completed the STARD reporting checklist. Available at https://dx.doi. org/10.21037/tp-21-381

Data Sharing Statement: Available at https://dx.doi. org/10.21037/tp-21-381

Conflicts of Interest: All authors have completed the ICMJE uniform disclosure form (available at https://dx.doi. org/10.21037/tp-21-381). The authors have no conflicts of interest to declare.

Ethical Statement: The authors are accountable for all aspects of the work in ensuring that questions related to the accuracy or integrity of any part of the work are appropriately investigated and resolved. All procedures performed in this study involving human participants were in accordance with the Declaration of Helsinki (as revised in 2013). The study was approved by the Ethics Committee of Beijing Chaoyang Hospital (No.: 2019-19) and informed consent was taken from all the patients. 
Open Access Statement: This is an Open Access article distributed in accordance with the Creative Commons Attribution-NonCommercial-NoDerivs 4.0 International License (CC BY-NC-ND 4.0), which permits the noncommercial replication and distribution of the article with the strict proviso that no changes or edits are made and the original work is properly cited (including links to both the formal publication through the relevant DOI and the license). See: https://creativecommons.org/licenses/by-nc$\mathrm{nd} / 4.0 /$.

\section{References}

1. Rogozinski LE, Alverson BK, Biondi EA. Diagnosis and treatment of Mycoplasma pneumoniae in children. Minerva Pediatr 2017;69:156-60.

2. Vervloet LA, Vervloet VE, Tironi Junior M, et al. Mycoplasma pneumoniae-related community-acquired pneumonia and parapneumonic pleural effusion in children and adolescents. J Bras Pneumol 2012;38:226-36.

3. He ZF, Wu SX, Ma KZ, et al, Clinical effect of bronchofiberscope alveolar lavage in the treatment of severe pneumonia in children. Clinical Research and Practice 2020;5:94-5.

4. Lin YL, Hung KL, Lo CW. Mycoplasma pneumoniaeassociated encephalitis complicated by cerebral salt wasting syndrome. Clin Case Rep 2017;5:1830-3.

5. Mishra R, Cano E, Venkatram S, et al. An interesting case of mycoplasma pneumonia associated multisystem involvement and diffuse alveolar hemorrhage. Respir Med Case Rep 2017;21:78-81.

6. Liu J, Ren XL, Fu W, et al. Bronchoalveolar lavage for the treatment of neonatal pulmonary atelectasis under lung ultrasound monitoring. J Matern Fetal Neonatal Med 2017;30:2362-6.

7. Respiratory Branch of Pediatrics Society of Chinese Medical Association; Editorial Board of Chinese Journal of Applied Clinical Pediatrics. Expert consensus on diagnosis and treatment of mycoplasma pneumoniae pneumonia in children (2015 edition). Chinese Journal of Applied Clinical Pediatrics 2015;30:1304-8.

8. Sa RN, Wang AQ, Li XA, et al. Clinical study of bronchoscopy lavage treatment for severe mycoplasma pneumoniae pneumonia in children. China Journal of Endoscopy 2020;26:79-85.

9. Yang M, Yang DH, Yang X, et al. Efficacy of bronchoalveolar lavage and its influence factors in the treatment of Mycoplasma pneumoniae pneumonia with atelectasis. Zhonghua Er Ke Za Zhi 2018;56:347-52.

10. Liu J, Fu W, Ao B, et al. Bronchoalveolar lavage for the treatment of neonatal pulmonary atelectasis under lung ultrasound monitoring in 32 cases. Chinese Journal of Perinatal Medicine 2017;20:213-8.

11. Reissig A, Copetti R, Mathis G, et al. Lung ultrasound in the diagnosis and follow-up of community-acquired pneumonia: a prospective, multicenter, diagnostic accuracy study. Chest 2012;142:965-72.

12. Xu SS, Guo LF, Wu Y, et al. Clinical features and epidemiological analysis of Mycoplasma pneumoniae pneumonia in children. Chinese Journal of Nosocomiology 2017;27:3307-10.

13. Luo YL, Chen ZR, Zhang XX, et al. Changes in epidemiology and clinical manifestations of mycoplasma infection in children in Suzhou during 2005-2014. Chinese Journal of Hemorheology 2015;25:480-5, 496.

14. Zhang H, Shang YX. Value of fiberoptic bronchoscopy in the diagnosis and treatment of refractory mycoplasma pneumoniae pneumonia in children. Chinese Journal of Practical Pediatrics 2019;34:504-7.

15. Leong MA, Nachajon R, Ruchelli E, et al. Bronchitis obliterans due to Mycoplasma pneumonia. Pediatr Pulmonol 1997;23:375-81.

16. Wang $\mathrm{X}, \mathrm{He} \mathrm{H}$, Zheng J, et al. A comparison of efficacy and safety of complementary and alternative therapies for severe mycoplasma pneumonia in children: a protocol for systematic review and meta-analysis. Medicine (Baltimore) 2021;100:e23959.

17. Liu J. Review and prospect of pediatric pulmonary ultrasound in China. Chinese Journal of Practical Pediatrics 2019;34:753-6.

18. Song YH. Incidence and prognosis of mycoplasma pneumoniae pneumonia complicated with atelectasis in children. Heilongjiang Medicine Journal 2015;28:629-30.

19. Hu YM, Jiang ZF, Shen KL, et al. editors. Zhu Futang practice of pediatrics. Version 8. Beijing: People's Medical Publishing House, 2015:1204-5.

20. Cai Q. Effect of bronchofiberoptic alveolar lavage on mycoplasma pneumoniae pneumonia complicated with atelectasis in children. The Medical Forum 2020;24:2270-2.

21. Ma X. Nursing effect of bronchiectasis treated with alveolar lavage assisted by fiberoptic bronchoscope. Laboratory Medicine and Clinic 2017;14:313-5.

22. Shi Y, Huang Y, Zhang TT, et al. Chinese guidelines for the diagnosis and treatment of hospital-acquired pneumonia and ventilator-associated pneumonia in adults 
(2018 Edition). J Thorac Dis 2019;11:2581-616.

23. Jin F, Wang H, Li Q, et al. Expert consensus for diagnosis and treatment using medical thoracoscopy in China. J

Cite this article as: Li S, Zhang QL, Guo RJ, Lv XZ, Yang X. Quantitative evaluation and significance of ultrasound in bronchoalveolar lavage for lung consolidation in children with severe mycoplasma pneumonia. Transl Pediatr 2021;10(9):2325-2334. doi: 10.21037/tp-21-381
Thorac Dis 2020;12:1799-810.

(English Language Editor: A. Kassem) 


\section{Supplementary}

Table S1 Logistic analysis results

\begin{tabular}{|c|c|c|c|c|c|c|c|c|}
\hline \multirow{2}{*}{ Contents } & \multicolumn{4}{|c|}{ Univariate } & \multicolumn{4}{|c|}{ Multivariate } \\
\hline & OR & Lower & Upper & $\mathrm{P}$ & OR & Lower & Upper & $\mathrm{P}$ \\
\hline Gender & -0.61313 & -0.01542 & 0.008348 & 0.545328188 & & & & \\
\hline Age & 0.529852 & -0.01596 & 0.027009 & 0.600888991 & & & & \\
\hline LY\% [20-50] & -0.39776 & -0.01795 & 0.012113 & 0.693825 & & & & \\
\hline NST [0-5\%] & -0.37258 & -0.02671 & 0.018491 & 0.712268 & & & & \\
\hline CRP [0-10] & 0.916583 & -0.00868 & 0.022731 & 0.367189 & & & & \\
\hline PTC & 0.078198 & -1.83372 & 1.979276 & 0.938227 & & & & \\
\hline Lung consolidation area before lavage & 3.381789 & 0.006447 & 0.026255 & 0.002141 & -0.40564 & -0.03363 & 0.022585 & 0.688603 \\
\hline $\begin{array}{l}\text { Area of pulmonary consolidation } \\
\text { immediately after lavage }\end{array}$ & 3.331948 & 0.006038 & 0.025309 & 0.002433 & 0.498701 & -0.0204 & 0.033394 & 0.62253 \\
\hline $\begin{array}{l}\text { Area of lung consolidation } 1 \text { days after } \\
\text { lavage }\end{array}$ & 1.972081 & -0.00053 & 0.026682 & 0.058924 & -0.41731 & -0.02463 & 0.016399 & 0.68069 \\
\hline $\begin{array}{l}\text { Area of lung consolidation } 3 \text { days after } \\
\text { lavage }\end{array}$ & 0.616676 & -0.0127 & 0.023611 & 0.542614 & & & & \\
\hline $\begin{array}{l}\text { Area of lung consolidation } 7 \text { days after } \\
\text { lavage }\end{array}$ & -1.80025 & -0.04681 & 0.003018 & 0.082604 & -1.95715 & -0.03463 & 0.001051 & 0.063755 \\
\hline
\end{tabular}

OR, odds ratio; Cl, confidence interval; NE\%, neutrophil\%; LY\%, lymphocyte\%; NST, neutrophilic stab granulocyte; CRP, C-reaction protein; PCT, procalcitonin. 